\title{
Growth, magnetism and ferromagnetic thickness gap in Fe films on the W(111) surface
}

\author{
Qiang Wu, ${ }^{1}$ R. Zdyb, ${ }^{2}$ E. Bauer, ${ }^{3}$ and M. S. Altman ${ }^{1, *}$ \\ ${ }^{1}$ Department of Physics, Hong Kong University of Science and Technology, Clear Water Bay, Kowloon, Hong Kong \\ ${ }^{2}$ Institute of Physics, Marie Curie-Skłodowska University, Lublin, Poland \\ ${ }^{3}$ Department of Physics, Arizona State University, Tempe, Arizona, USA
}

(Received 30 November 2012; published 20 March 2013)

\begin{abstract}
The growth, structure, and magnetism of Fe films on the W(111) surface were investigated using low energy electron microscopy (LEEM) and diffraction (LEED), spin polarized LEEM (SPLEEM), and work function measurements. In contrast to an earlier report that Fe grows with fcc structure following initial pseudomorpic layer growth, we observe no evidence of the formation of fcc Fe over the entire thickness range studied, up to 18 monolayers (ML). Observations are instead consistent with the formation of a well-ordered, laterally (tensile) strained bcc Fe layer that gradually relaxes vertically and develops increasing disorder with increasing thickness. Ferromagnetic order appears at $6 \mathrm{ML}$, but surprisingly vanishes at $8 \mathrm{ML}$, and reappears just as suddenly at 9 ML during Fe deposition at room temperature. Ferromagnetism between 6 and 8 ML also vanishes at only 5 deg above room temperature. The magnetization direction of a monodomain structure remains constant before and after the ferromagnetic thickness gap at 8-9 ML until the formation of a multidomain structure at about $12 \mathrm{ML}$. Variations of exchange asymmetry in spin-polarized elastic electron scattering are also observed with increasing film thickness, particularly above $12 \mathrm{ML}$, that indicate changes in the spin-polarized electron band structure above the vacuum level. The evolution of magnetism and exchange asymmetry with increasing thickness and the appearance of the ferromagnetic gap are attributed to structural and morphological changes in the strained $\mathrm{Fe}$ layer, which eventually lead to a relaxed although highly disordered bcc Fe layer.
\end{abstract}

DOI: $10.1103 /$ PhysRevB.87.104410

PACS number(s): 75.70.Ak, 68.55.A-, 75.70.Kw, 68.37.Nq

\section{INTRODUCTION}

The magnetic spin configurations of fcc $\mathrm{Fe}(\gamma-\mathrm{Fe})$ have attracted considerable attention over the years. ${ }^{1-8}$ The fact that fcc $\mathrm{Fe}$ is only stable in bulk in a temperature range, $1183-1663 \mathrm{~K}$, where magnetic ordering is not expected to occur, e.g., above the Curie temperature exhibited by bcc Fe $(\alpha-\mathrm{Fe})$, has not dampened this interest. This issue has, in fact, stimulated experimental efforts to stabilize fcc Fe artificially at low temperature, with intriguing results. Experimentally, the magnetic ground state of fcc Fe precipitates stabilized with small amounts of $\mathrm{Co}$ at the $\mathrm{Cu}$ lattice constant in a $\mathrm{Cu}$ matrix was found to have a noncollinear spin-spiral configuration. ${ }^{5}$ This discovery was followed by numerous theoretical investigations of noncollinear spin configurations using increasingly sophisticated methods. ${ }^{6-8}$ Not only was the spin-spiral ground state confirmed, it was also found that the magnetic structure and moments of fcc Fe depend sensitively upon changes of lattice constant from equilibrium, analogous to earlier predictions for simpler collinear spin configurations. $^{1-4}$

A well-known approach to stabilize fcc Fe at low temperature is to grow Fe films on fcc substrates, particularly $\mathrm{Cu}(100)$ and $\mathrm{Cu}(111)$ substrates due to the close lattice match with fcc $\mathrm{Fe}^{9-14}$ However, the formation of bcc-like inclusions in $\mathrm{Fe}$ films on $\mathrm{Cu}$ suggests an origin of ferromagnetism or at least a contributing factor to the controversial magnetic behavior in these systems. ${ }^{15-17}$ Interestingly, it was reported that fcc Fe films can also be stabilized on the bcc W(111) substrate surface. ${ }^{18}$ It was observed using low energy electron diffraction (LEED) that Fe grows pseudomorphically at room temperature up to a thickness that was identified as 2 monolayers. The presence of superstructure diffraction spots between 2 and 4 monolayers was interpreted as evidence of an fcc film that is laterally contracted by $1 \%$ compared to bulk Fe, assuming a bulk lattice constant of $3.61 \AA$, with its [1 12$]$ direction parallel to the substrate $[1 \overline{1} 0]$ direction. The presence of an fcc film above 4 monolayers with the same orientation but with $1.3 \%$ lateral lattice expansion compared to bulk fcc Fe was also inferred from LEED observations. ${ }^{18}$ Formation of pseudomorphic (ps) bcc $\mathrm{Fe} / \mathrm{W}(111)$ requires close to $10 \%$ tensile strain of the Fe film. On the other hand, the lattice constant of the $\mathrm{W}(111)$ surface $(4.47 \AA)$ is very close to the next-nearest neighbor distance in the fcc $\mathrm{Fe}(111)$ layer (4.42 ̊).

Some insight about the magnetic properties of $\mathrm{Fe} / \mathrm{W}(111)$ is provided by observations of spin polarized field emission (SPFE) from Fe-coated W(111) tips. ${ }^{19}$ Somewhat more is known about magnetism of films on W(111) from similar studies of SPFE from Co-coated W(111) tips ${ }^{19,20}$ and from studies of Co films on a macroscopic W(111) surface. ${ }^{21,22}$ In the present work we extend these earlier studies by investigating the room temperature growth and magnetic properties of Fe films on a macroscopic W(111) surface using low energy electron microscopy (LEEM), spin polarized LEEM (SPLEEM), LEED, and work function measurements. The aim of this work is to reassess the formation of fcc Fe films and to explore the sensitivity of its magnetic properties to the change of lattice constant that purportedly occurs with increasing film thickness.

\section{EXPERIMENTAL DETAILS}

The W(111) substrate was cleaned using standard procedures of annealing in oxygen at about $1400 \mathrm{~K}$ to remove carbon, and flashing up to about $2200 \mathrm{~K}$ to remove oxygen. The sample was heated by electron bombardment and its 
temperature was measured using a W3\%/Re-W25\%/Re thermocouple. It was found that the magnetic order of the $\mathrm{Fe}$ films investigated here depended sensitively upon temperature in a very narrow range spanning a few degrees above room temperature. Therefore, several additional measures were taken in order to study this behavior reliably. First of all, the sample temperature measurement was supplemented by measurements of room air temperature and the vacuum chamber temperature at strategic positions on the sample manipulator and near the Fe deposition source. These measurements were performed in order to eliminate the chance that the sample temperature measurement was offset due to a cold or warm thermocouple junction, which could not be measured directly. The time sequencing of the cleaning sample flash to high temperature and cool down to room temperature was strictly controlled in order to assure reliable and reproducible initial conditions for each experiment. Sample temperature was measured with $0.001 \mathrm{mV}$ thermocouple resolution, corresponding to $0.09 \mathrm{~K}$ temperature resolution near room temperature. Temperatures slightly above room temperature were achieved by mild radiative heating by a filament behind the sample. Careful calibration of the sample heating filament current with $0.01 \mathrm{~A}$ resolution against the thermocouple reading indicated a reproducible sample temperature change of $0.35 \mathrm{~K} / 0.01 \mathrm{~A}$ in the temperature range of the studies reported here. Until all of these measures were taken, the results did not reproduce well. However, once these procedures were implemented, the sensitive temperature dependence of the magnetic behavior could be seen more clearly.

Prior to experiments on $\mathrm{W}(111)$, the Fe deposition rate was calibrated by monitoring the growth of 1 ps Fe monolayer (ML) on the $\mathrm{W}(110)$ surface, corresponding to $1.42 \times$ $10^{15}$ atoms $/ \mathrm{cm}^{2}$. The completion of the first ps Fe ML on $\mathrm{W}(110)$ is easily identified in LEEM imaging. The deposition rate on $\mathrm{W}(110)$ was then converted to the deposition rate on $\mathrm{W}(111)$ by scaling with the atomic density of a W(111) layer, $5.7 \times 10^{14}$ atoms $/ \mathrm{cm}^{2}$. This defines the "geometric monolayer" which is conventionally referred to simply as monolayer (ML). The deposition rates employed in our investigations were chosen in the range $0.12-0.15 \mathrm{ML} / \mathrm{min}$. Film thickness on bcc(111) surfaces has also been expressed in the literature in units of the "physical monolayer" (PML), which is equivalent to three geometric ML. ${ }^{23}$ One PML is required to completely cover all substrate atoms that are exposed in the surface layer, first and second subsurface layers of the bcc(111) surface. To avoid confusion, film thickness is expressed here in geometric ML units, not PML, except where explicitly stated. An additional reason for touching on this point will become clear in the discussion of Sec. III A below.

The deposited Fe film thickness is determined by the product of the deposition rate and deposition time, which was controlled with $1 \mathrm{~s}$ precision corresponding to $2 \times$ $10^{-3} \mathrm{ML}$. The deposition rate was found to be reproducible within $2 \%$ over the span of many experiments. Furthermore, a more precise measure of the deposition rate was provided during each deposition by periodic intensity oscillations that occur due to layer-by-layer growth (Fig. 1), corroborated by the reproducible onset of magnetic signal in magnetic measurements. It is estimated that these measures facilitated control of Fe film thickness to better than 0.05 ML.

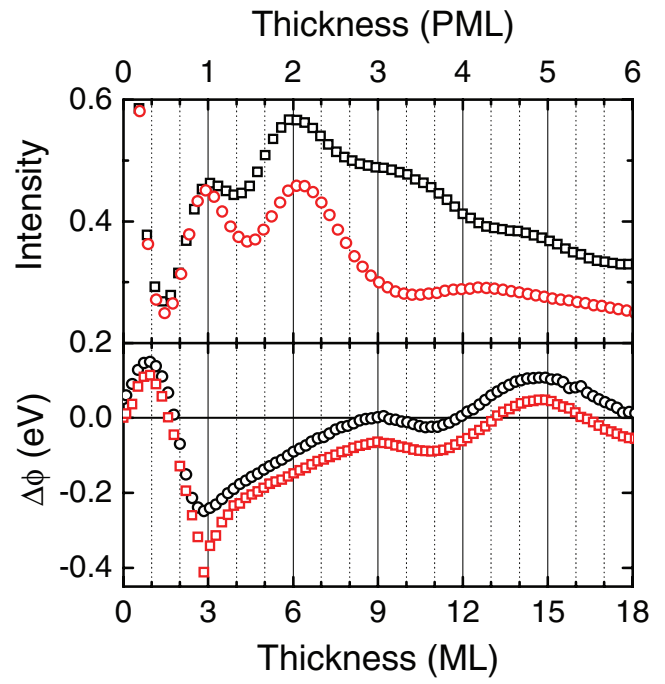

FIG. 1. (Color online) (Top) Normalized LEEM image intensity at $16 \mathrm{eV}(\bigcirc)$ and $18 \mathrm{eV}(\square)$ and (bottom) work function change measured by mode (i) ( $\square$ ) and mode (ii) ( $\bigcirc$ ) (see text) vs film thickness during Fe deposition on W(111) at room temperature. Mode (ii) results are shifted by $+0.05 \mathrm{eV}$ for clarity.

LEEM, LEED, and work functions measurements were carried out in a conventional low energy electron microscope (LEEM). Magnetic imaging measurements were carried out in a spin polarized LEEM (SPLEEM). The base pressures in the SPLEEM and LEEM were $\sim 1.8 \times 10^{-10}$ and $1.5 \times$ $10^{-10}$ Torr, respectively. The pressure rose respectively to $2.0 \times 10^{-10}$ and $2.5 \times 10^{-10}$ Torr during Fe deposition. The imaging and diffraction capabilities and principles of LEEM and SPLEEM have been discussed previously. ${ }^{24,25}$ Specific conditions for this experiment are the inelastic mean free path (IMFP) in Fe, which is $5 \AA$ in the energy range used, ${ }^{26}$ and an "effective" transfer width of $2 \pi / F W H M=75 \AA$ as deduced from the full width at half maximum (FWHM) of the (00) diffraction spot at the energies used for imaging. The former determines the information depth, the latter is inversely related in a nontrivial way via the electron source characteristics (source extension, energy spread) to the smallest lateral dimension that can be resolved in imaging ${ }^{27}$ and to sample perfection. Compared to the transfer width of $\sim 300 \AA$ achieved with the LEEM on atomically flat surfaces, ${ }^{25,28}$ the small effective transfer width observed here is due mainly to the roughness of the $\mathrm{W}(111)$ surface, which appears structureless in LEEM.

The conventional LEEM imaging and diffraction capabilities are augmented by magnetic sensitivity when a spin polarized electron beam is used to illuminate the sample in SPLEEM. Magnetic measurements are performed by acquiring images with oppositely polarized incident beams, called spin-up and spin-down images. The exchange asymmetry is defined as $A_{\mathrm{ex}}=(1 / P)\left(I_{\uparrow}-I_{\downarrow}\right) /\left(I_{\uparrow}+I_{\downarrow}\right)$, where $I_{\uparrow}$ and $I_{\downarrow}$ represent the reflected intensities for spin-up and spin-down images, and $P$ is the degree of spin polarization of the incident beam. Image subtraction in the numerator eliminates nonmagnetic information that is contained in conventional unpolarized LEEM images and leaves features that originate exclusively in the magnetism of the sample. Division by the 
image sum yields an asymmetry image in which intensities are proportional to the component of the local magnetization vector that lies along the incident beam polarization direction. Although the proportionality is energy dependent, the magnetization direction can still be evaluated by adjusting the incident beam spin polarization direction using a spin rotator. ${ }^{29}$ This allows complete characterization of the sample magnetization direction in the in- and out-of-plane directions and tilted directions in between.

The work function change during $\mathrm{Fe}$ deposition was measured using the retarding field method by monitoring the transition from LEEM imaging to mirror electron microscopy (MEM) mode. ${ }^{30,31}$ The transition from LEEM to MEM is identifiable by a sharp rise of image intensity as the incident electron energy is reduced. Two measurement modes were used to determine work function changes: (i) repeatedly recording the MEM to LEEM transition intensity curves during Fe deposition and plotting the shifting voltage at a fixed fraction $(50 \%)$ of the saturation MEM intensity, and (ii) continuously recording the image intensity at fixed energy in the MEM to LEEM transition region during deposition. Intensity changes measured by mode (ii) are proportional to work function change by the slope of the intensity curve at the transition from LEEM to MEM. The accuracy of the work function determination in this mode may be undermined by possible nonlinearity of the intensity curve, but this mode gives the general trend and is in principle convenient for rapid measurements. Excellent agreement of results obtained by the two modes (Fig. 1) confirms the validity of the latter.

\section{EXPERIMENTAL RESULTS}

\section{A. Growth}

In order to make a connection to the work reported in Ref. 18, the meaning of the monolayer coverage unit in that work, i.e., geometric (ML) or physical (PML) monolayer, must first be clarified. We have done this by measuring work function changes and performing complementary LEEM imaging experiments during Fe deposition at room temperature. The work function change determined here, in particular, can be compared directly to the results of similar measurements that were reported in Ref. 18. No spatially distinct features were observed in bright field LEEM images during Fe deposition. The image intensity instead was spatially uniform and exhibited oscillations as Fe thickness was increased (Fig. 1). Two distinct intensity peaks were observed at equal time intervals during deposition at many imaging energies. According to the rate calibration of the deposition source, these peaks occur at film thicknesses of $3 \mathrm{ML}$ (1 PML) and $6 \mathrm{ML}$ (2 PML). This behavior is similar to behavior that was observed previously during growth of Co on $\mathrm{W}(111) .^{21}$

Work function changes that were recorded during $\mathrm{Fe}$ deposition are shown in Fig. 1. The work function initially increases to a maximum at about $1 \mathrm{ML}$ (0.33 PML), according to the deposition rate calibration. Then, it decreases to a deep minimum at $3 \mathrm{ML}$ (1 PML). This is the same film thickness that the first LEEM image intensity peak is observed (Fig. 1). Thereafter, the work function increases to a second peak almost exactly at $9 \mathrm{ML}$ (3 PML). These changes and those that follow at larger thickness are in very good agreement with work function changes that were reported previously in Ref. 18, provided we identify the monolayer unit in that work to be the PML. This comparison resolves the ambiguity about the film thickness scale in Ref. 18 and also establishes a clear link between the two investigations. However, it is also very interesting to note that the previous study reported a work function increase from its minimum at $3 \mathrm{ML}$ to the maximum at $9 \mathrm{ML}$ in two stages, initially by a weak increase to a constant value between about 4 and $6 \mathrm{ML}$ followed by a sharp rise to 9 ML. ${ }^{18}$ This behavior is in contrast to the continuous smooth increase after the sharp initial rise seen reliably between 3 and $9 \mathrm{ML}$ here. The origin of this difference may lie in the deposition method. Deposition was carried out by incremental doses between measurement intervals in the earlier work, while measurements were carried out during continuous deposition here. An interpretation of the different work function behavior and their relationships to the growth mode are discussed further in Sec. IV.

\section{B. Structural evolution}

In contrast to earlier LEED observations, ${ }^{18}$ we find no evidence of the superstructure diffraction spots that were interpreted previously as evidence of an fcc Fe film. The possible origins of this discrepancy are discussed in Sec. IV. Furthermore, we observe that the integer order diffraction spots do not shift laterally with increasing Fe film thickness. This observation indicates that the lateral periodicity does not change after the formation of the initial pseudomorphic layer, which according to Ref. 18 is stable only up to $6 \mathrm{ML}$ (corrected value).

In order to shed light on vertical atomic arrangements of the Fe film during growth, we have measured the bright field LEEM image intensity as a function of energy, i.e., the LEEM $I(V)$, during Fe deposition. The bright field LEEM image is formed from the (00) diffraction beam, corresponding to the diffraction condition with no parallel momentum transfer. This diffraction condition gives greatest sensitivity to vertical structural arrangement. The energy was repeatedly scanned through a $20 \mathrm{eV}$ range around the first intensity peak at lowest energy during uninterrupted $\mathrm{Fe}$ deposition at room temperature. LEEM $I(V)$ curves shown in Fig. 2 exhibit peaks whose positions depend upon thickness. We assign the experimental peak from the clean $\mathrm{W}$ surface at $16.0 \mathrm{eV}$ to the bulk-forbidden (111) Bragg peak, which is expected from simple kinematical considerations to be located at $11.29 \mathrm{eV}$. Similarly, the peak at the largest thickness shown in Fig. 2 at $18.0 \mathrm{eV}$ can be assigned to the bulk-forbidden (111) Bragg peak of bulk bcc Fe, which is expected at $13.69 \mathrm{eV}$. The experimental energy scale in this figure is referenced to the instrument power supply, which is offset by a constant value from the Fermi level. The differences between the experiment and expected peak positions (4.71 eV for $\mathrm{W}$ and $4.31 \mathrm{eV}$ for $\mathrm{Fe}$ ) are contributed to by the work function of the $\mathrm{LaB}_{6}$ cathode $(2.7 \mathrm{eV})$ and by the self-correction energy, ${ }^{32}$ which is of the order of 1 to several eV, e.g., $1.6 \mathrm{eV}$ for $\mathrm{Ni}^{33}$

Similarly, the peak developing strongly above 1 ML initially at about $25.0 \mathrm{eV}$ is consistent (with similar offset) with the bulk-forbidden (111) Bragg peak of a bcc $\mathrm{Fe}(111)$ layer that is 

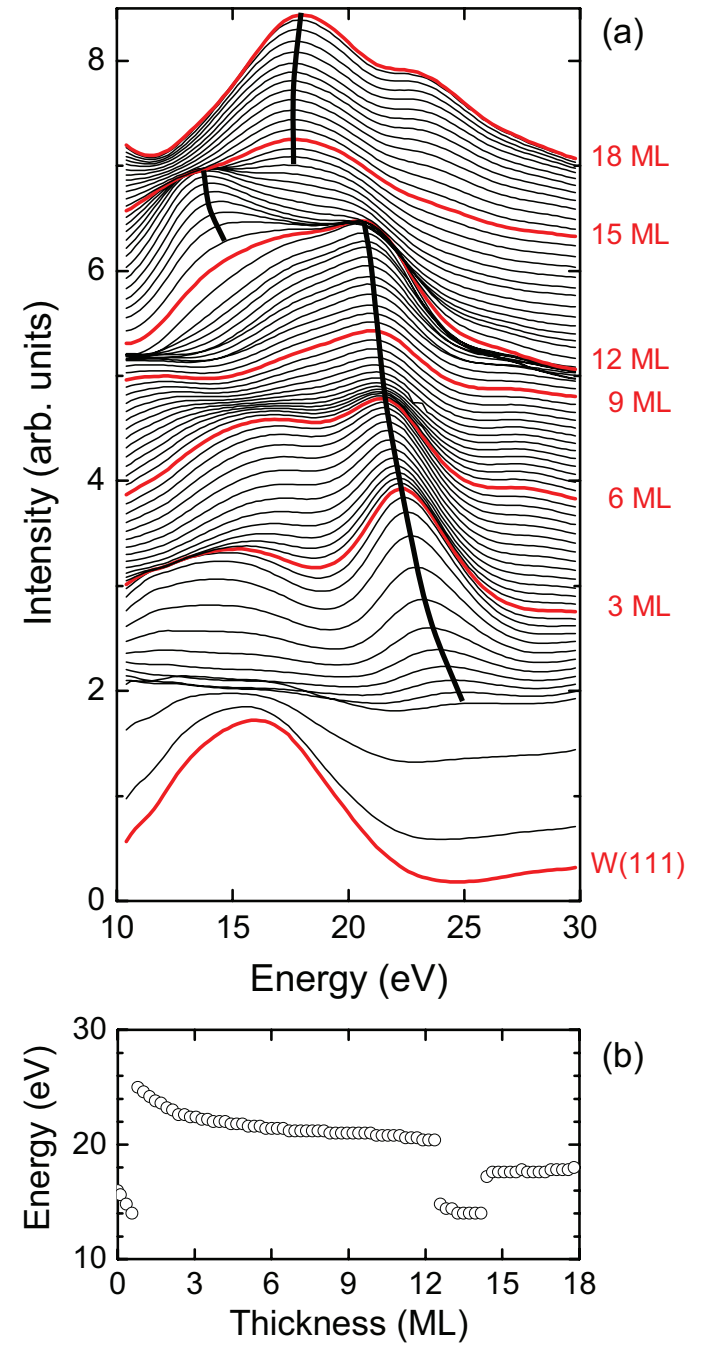

FIG. 2. (Color online) (a) LEEM $I(V)$ curves for the clean and Fe covered W(111) surface, measured during continuous Fe deposition at room temperature, are shown for film thickness from 0 to $18 \mathrm{ML}$ at roughly $0.21 \mathrm{ML}$ intervals. The data at $3 \mathrm{ML}$ intervals are indicated by thick (red) curves. The curves are shifted vertically and intensity is scaled at larger thickness for clarity. (b) The position of the dominant intensity peaks are indicated by solid lines and are plotted vs energy. The energy scale is referenced to the instrument power supply (see text).

vertically contracted and laterally (tensile) strained to lattice match to the W(111) substrate, ${ }^{34}$ which is expected at $19.98 \mathrm{eV}$. An fcc $\mathrm{Fe}(111)$ layer $^{34}$ can unambiguously be ruled out because its first allowed Bragg peak, i.e., (111), is expected at a considerably lower energy of $8.63 \mathrm{eV}$. The dominant $\mathrm{Fe}$ intensity peak shifts continuously from $25.0 \mathrm{eV}$ at $1 \mathrm{ML}$ to $20.4 \mathrm{eV}$ at 12.4 ML, suggesting that the vertical contraction of the film diminishes with increasing thickness. As the thickness approaches 12.4 ML, a weak peak appears at the low energy shoulder of the dominant peak. Gradual intensity redistribution between these two peaks finally at 12.6 ML causes the lower energy peak, now at $14.8 \mathrm{eV}$, to dominate. This peak then drifts slightly lower to $14.0 \mathrm{eV}$ at $14.2 \mathrm{ML}$, whereupon a similar continuous intensity redistribution gives rise at 14.4 ML to the dominance of a peak at $17.4 \mathrm{eV}$. This peak shifts slowly
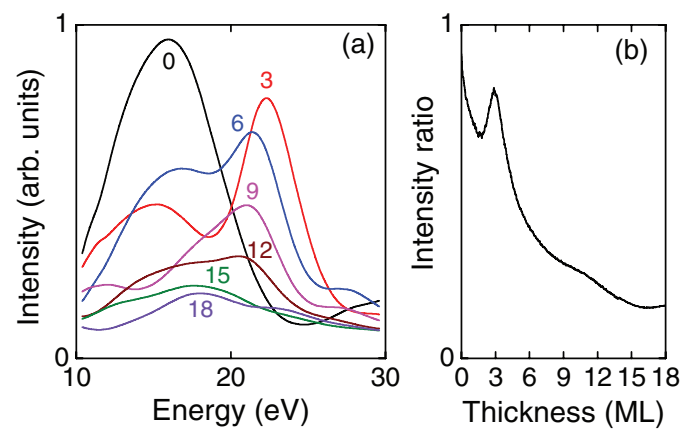

FIG. 3. (Color online) (a) As-measured LEEM $I(V)$ curves for clean W(111) and indicated Fe thicknesses. (b) Ratio of elastically scattered intensity in the diffraction spots to the background intensity integrated over all reciprocal space at incident energy $22 \mathrm{eV}$.

upward to $18.0 \mathrm{eV}$ at $18 \mathrm{ML}$. Interpretations of these changes will be discussed in more detail in Sec. IV.

The $I(V)$ curves in Fig. 2 have been shifted and their intensity enhanced at the largest thicknesses in order to bring out the peak positions more clearly. The as-measured intensities are shown to scale in Fig. 3(a) at $3 \mathrm{ML}$ intervals and the ratio of the integrated intensities of all diffracted peaks [including the (00) peak] to the background integrated over reciprocal space is shown as a function of thickness in Fig. 3(b). The strong peak in Fig. 3(a) for 3 ML Fe illustrates the high perfection of the initial pseudomorphic film but the peaks at larger thickness seen in the figure are strongly suppressed due to growing disorder. The decreasing ratio in Fig. 3(b) gives the true picture of the loss of order with increasing film thickness.

\section{Magnetic properties}

The spontaneous sample magnetization was probed by measuring the magnetic exchange asymmetry in SPLEEM images as a function of film thickness, incident electron energy, and incident electron spin polarization direction. SPLEEM magnetic asymmetry images obtained at two incident electron energies during uninterrupted $\mathrm{Fe}$ deposition at room temperature are shown for selected film thicknesses in Fig. 4. These images were acquired with incident beam spin polarization orientation selected for sensitivity to in-plane magnetization along its easy axis direction. This corresponds to the azimuthal direction $\phi=80^{\circ}$ with respect to the instrument reference direction. These images reveal single domain structure over a length scale that exceeds the image field of view up to about $12 \mathrm{ML}$ thickness. A multidomain structure becomes faintly visible at $13 \mathrm{ML}$ thickness at low incident electron energy $(\sim 1 \mathrm{eV})$ and becomes more obvious with increasing thickness.

The variation of the magnetic asymmetry with increasing film thickness during deposition at room temperature reveals that the onset of magnetization occurs at $6 \mathrm{ML}$ (Fig. 5), corresponding roughly to the number of atoms in two closedpacked Fe layers. This onset is mainly determined by finite size effects that suppress the Curie temperature in thin films, ${ }^{35}$ and is similar to onset thicknesses of ferromagnetic order in Fe films on other substrate surfaces ${ }^{36-38}$ and in Co films on the $\mathrm{W}(111)$ surface $^{21}$ at room temperature. After the onset of magnetization, the variation of asymmetry exhibits different complex behavior at different imaging energies 


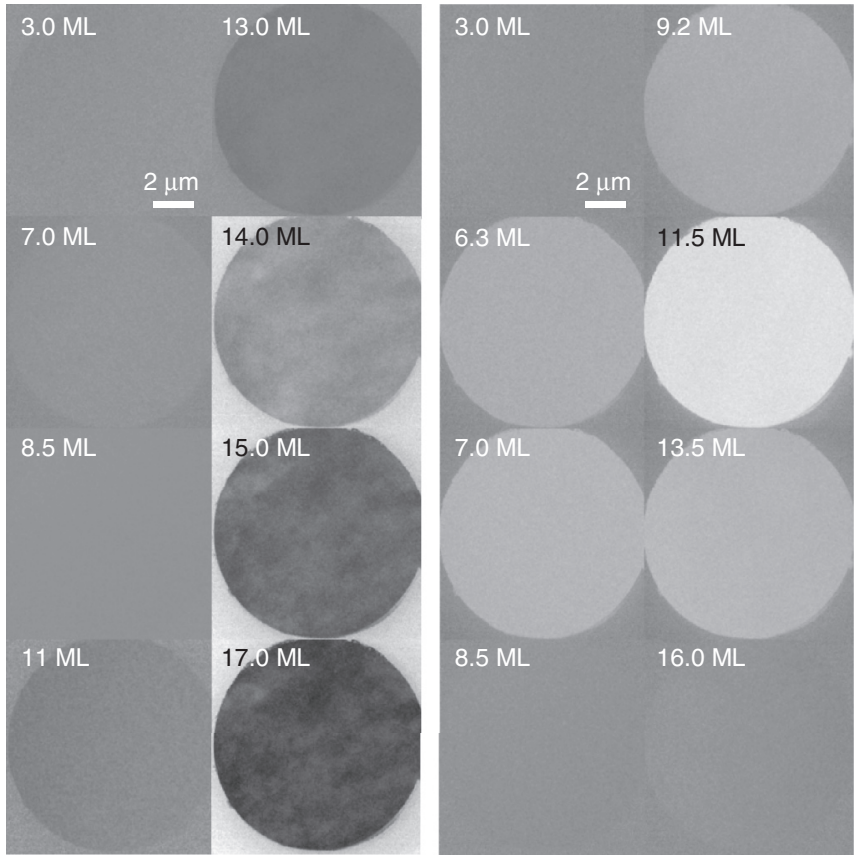

FIG. 4. SPLEEM images obtained at incident energies (left) $1.4 \mathrm{eV}$ and (right) $6.8 \mathrm{eV}$ during Fe deposition at room temperature are shown for selected Fe film thicknesses. The incident spin polarization direction probes in-plane sample magnetization along the easy axis corresponding to the azimuthal direction $\phi=80^{\circ}$ with respect to the instrument reference direction. Images on the left at $14 \mathrm{ML}$ and above have been processed to enhance the weak domain contrast at $1.4 \mathrm{eV}$.

(Fig. 5). This contrasts sharply with the monotonic increase of asymmetry that is observed with increasing film thickness in the Co/W(111) system. ${ }^{21}$ Most surprisingly, the asymmetry for $\mathrm{Fe} / \mathrm{W}(111)$ vanishes abruptly at $8 \mathrm{ML}$ at room temperature and reappears just as suddenly at $9 \mathrm{ML}$ at all of the selected energies. Measurements of asymmetry as functions of incident energy $A_{\mathrm{ex}}(E)$ and the incident spin polarization direction $A_{\mathrm{ex}}(\phi)$ (Fig. 6) confirm the complete loss of magnetic signal at all energies and in-plane and out-of-plane directions in this thickness range.

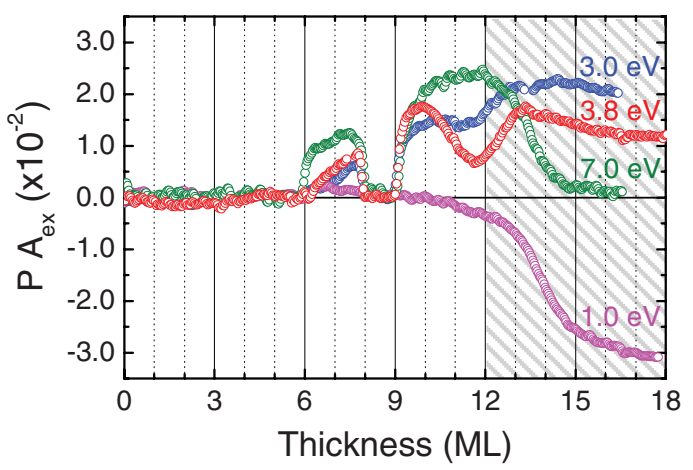

FIG. 5. (Color online) SPLEEM magnetic asymmetry as a function of Fe film thickness at the indicated incident energies, measured at $298.8 \mathrm{~K}$ for the in-plane direction at $\phi=80^{\circ}$. A ferromagnetic thickness gap is observed at all energies between 8 and 9 ML. The multidomain structure appears in the thickness range above $12 \mathrm{ML}$.

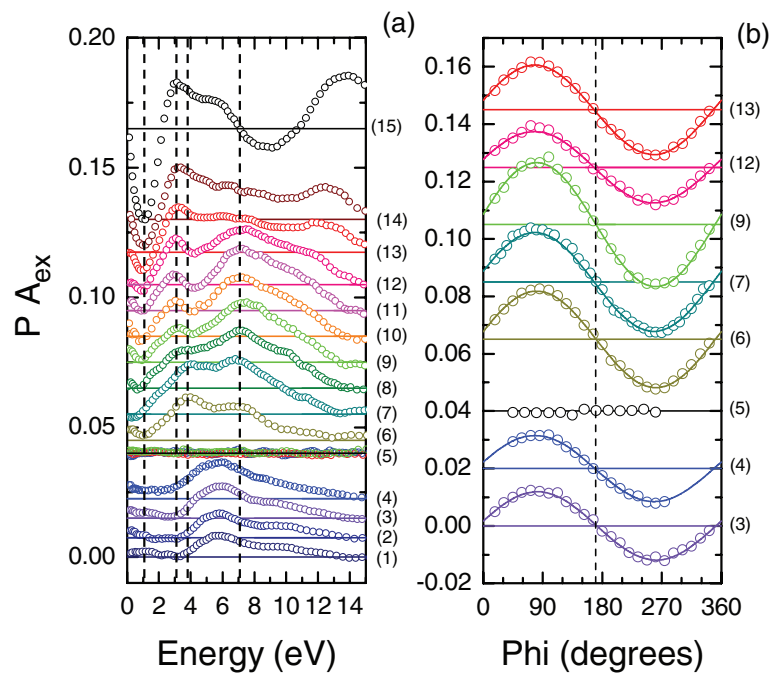

FIG. 6. (Color online) SPLEEM magnetic asymmetry as a function of (a) incident electron energy relative to the vacuum level and (b) in-plane azimuthal spin polarization direction $(\phi)$ are shown for incrementally thicker Fe films: (1) 6.29 , (2) 6.45, (3) 6.99, (4) 7.49 , (5) 8.50, , (6) 9.20, (7) 10.0, (8) 10.69, (9) 10.85, (10) 11.07, (2) 11.36, (12) 12.18, , (13) 12.71, (14) 13.23, (15) $18.07 \mathrm{ML}$. The measurements in (a) probed in-plane magnetization along the easy axis $\left(\phi=80^{\circ}\right)$ for all thicknesses. Null results for the orthogonal in-plane $\left(\phi=170^{\circ}\right)$ and out-of-plane directions are shown for $8.5 \mathrm{ML}$ as well. The vertical dashed lines in (a) indicate the energies of the measurements shown in Fig. 5. In (b), measurements were made at incident energy $E=$ $7.0 \mathrm{eV}$. The curves are offset for clarity.

The sinusoidal dependence of asymmetry on in-plane azimuthal angle below the appearance of the multidomain structure at about 13 ML [Fig. 6(b)], except in the ferromagnetic thickness gap between 8 and $9 \mathrm{ML}$, indicates uniaxial magnetization. No evidence of an out-of-plane magnetization component is observed under any condition. Figure 6(a) also reveals that changes in the shape of the $A_{\mathrm{ex}}(E)$ curve occur with increasing film thickness. These changes underlie the complex variations of asymmetry that are detected at different fixed energies during film growth (Fig. 5). The first significant change is a transition from a single to double-peaked energy dependence on either side of the ferromagnetic thickness gap. The changes of asymmetry between about 12 and $15 \mathrm{ML}$ are particularly pronounced. This is the thickness range that the multidomain structure develops (Fig. 4) and that the occurrence of major structural changes is suggested by the $I(V)$ curves in Fig. 2.

In the course of these experiments, we observed that the strength of the asymmetry signal in the thickness range 68 ML, i.e., before the ferromagnetic thickness gap, depends very sensitively upon temperature. The results of systematic measurements of this effect during growth at fixed temperature and during temperature variations at fixed thickness are shown in Figs. 7 and 8, respectively. The magnetic asymmetry is observed in an increasingly narrow thickness range centered at $7 \mathrm{ML}$ during growth when the growth temperature is increased in small steps over a narrow range [Fig. 7(a)]. The magnitude of the asymmetry in this thickness range is similarly sensitive to these small temperature changes. The width of the thickness 

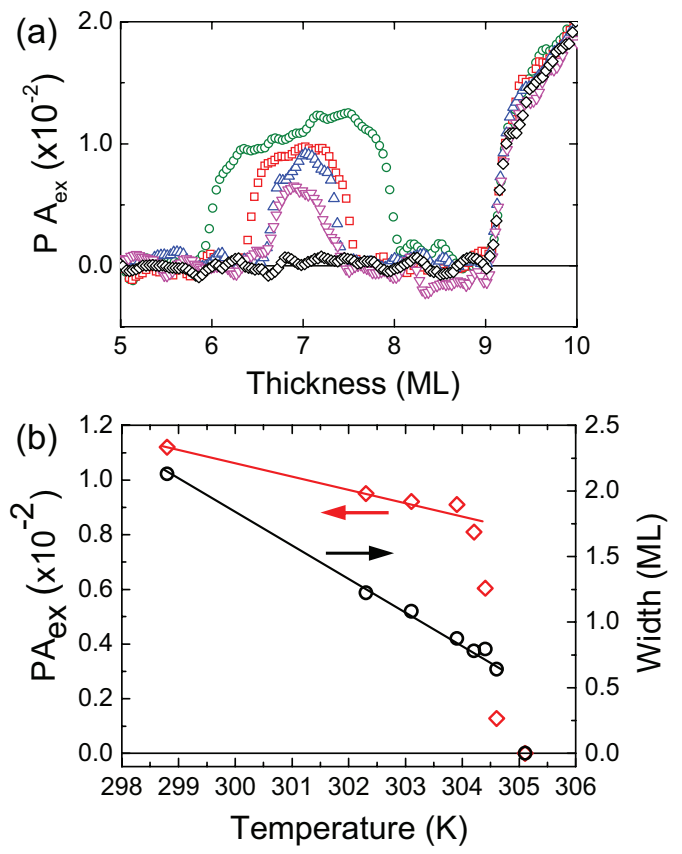

range in which asymmetry was detected and the magnitude of the asymmetry at $7 \mathrm{ML}$ thickness are shown vs deposition temperature in Fig. 7(b). A sharp cutoff in both quantities is observed at only $5 \mathrm{~K}$ above room temperature.

This effect was studied further by measuring the temperature dependence of the asymmetry at a few fixed film thicknesses in the range 6-8 ML (Fig. 8). In agreement with the behavior observed during deposition (Fig. 7), the asymmetry decreases sharply just above room temperature [Fig. 8(a)]. The critical temperature $T c$, defined as the temperature that asymmetry vanishes, is shown in Fig. 8(b). The error bars in this plot represent the reproducibility in several measurements at each thickness. While the increase of $T c$ up to $7.5 \mathrm{ML}$ is consistent with the trend expected due to finite size effects, ${ }^{35}$ the extrapolated trend exceeds the value and error bar at the largest thickness studied in this range (7.7 ML).

\section{DISCUSSION}

\section{A. Growth morphology and structure}

FIG. 7. (Color online) (a) SPLEEM magnetic asymmetry at $E=7.0 \mathrm{eV}$ is shown as a function of thickness during growth at several temperatures, $298.8 \mathrm{~K}(\bigcirc), 302.3 \mathrm{~K}(\square)$, $303.9 \mathrm{~K}(\triangle)$, $304.4 \mathrm{~K}(\nabla), 305.1 \mathrm{~K}(\diamond)$. (b) The width of the thickness range centered at $7 \mathrm{ML}$ in which asymmetry is observed during deposition $(\bigcirc)$ and the magnitude of asymmetry at $7 \mathrm{ML}(\diamond)$ are shown for different deposition temperatures.
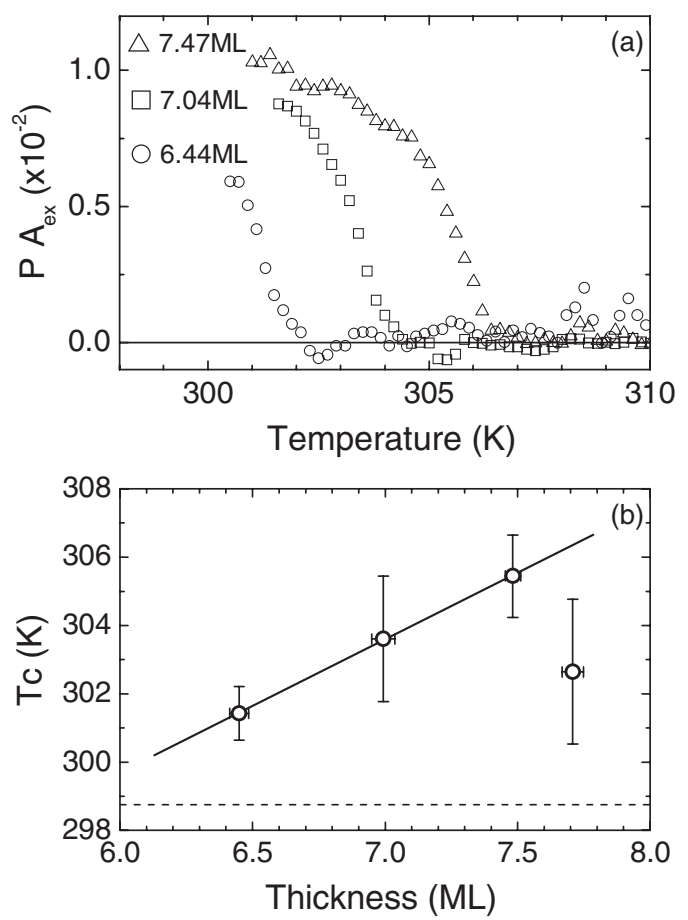

FIG. 8. (a) SPLEEM magnetic asymmetry at $E=7.0 \mathrm{eV}$ vs temperature is shown for the film thicknesses, 6.44 ML ( $\bigcirc)$, $7.04 \mathrm{ML}(\square), 7.47 \mathrm{ML}(\triangle)$. (b) The temperature $T c$, defined at the point that asymmetry vanishes during heating, is shown as a function of thickness. The horizontal dashed line indicates room temperature.

We observed diffraction intensity oscillations that are indicative of a distinct triple-monolayer (single PML) growth mechanism for Fe thicknesses below 9 ML (3 PML). Intensity measurements are not conclusive at greater thicknesses. Earlier Auger electron spectroscopy measurements suggested triple-layer growth up to about 15 ML (5 PML) "corrected" thickness, ${ }^{18}$ with the new understanding that the ML unit used previously is in fact the PML. Substantial agreement of work function changes observed here and earlier ${ }^{18}$ up to and beyond $18 \mathrm{ML}$ further establishes a link between the experiments. We attribute a difference of the work function change between 3 and $6 \mathrm{ML}$ noted in Sec. III A to the different deposition methods used in the two investigations, cumulative deposition by incremental doses interrupted by measurement intervals in Ref. 18 vs continuous deposition and measurement here. It is well-known that these two deposition methods can lead to different growth processes. In particular, continuous deposition can lead to metastable growth morphology, and possibly metastable structure as well. The formation of such metastable features might be avoided during cumulative deposition because growth interruption allows time for the system to relax to a thermodynamically stable state. We therefore believe that the shallow rise of the work function observed in Ref. 18 between 3 and $6 \mathrm{ML}$ (compared to the stronger rise shown in Fig. 1) represents the formation of an atomically rough surface consisting of 1-3 ML thick islands which are allowed to build up during the growth interruptions, e.g., through surface diffusion and coarsening. Growth interruptions could similarly open kinetic pathways to local atomic rearrangements that result in the superstructure above $6 \mathrm{ML}$ that was attributed to fcc Fe in Ref. 18, but was not detected here under continuous growth conditions.

In contrast to the previous report, ${ }^{18}$ we only observed a $(1 \times 1)$ LEED pattern with diminishing peak intensity and increasing background intensity (Fig. 3) up to the largest thickness studied in our investigations. Furthermore, the stationary positions of the integer-order diffraction spots indicated the absence of lateral relaxations as film thickness was increased continuously. The different LEED observations 
made here and previously ${ }^{18}$ could arise from the different deposition methods employed in the two investigations as noted above. Alternatively, the discrepancy could be caused by the influence of residual gas, such as $\mathrm{H}_{2}$ or $\mathrm{CO}$. The chamber pressure during the experiments described in Ref. 18 was in the upper $10^{-11}$ Torr range, ${ }^{39}$ slightly better than the current work. Notwithstanding the difficulty of comparing pressure measurements in different chambers, the slightly different vacuum condition does not seem to be significant enough to account for the starkly different diffraction results.

We also observed that the $I(V)$ curve of the $(00)$ beam changes significantly with increasing film thickness in the energy range of the first Bragg peak. These changes identify modifications of film structure in the vertical direction. From the peak shifts seen in Fig. 2 and the peak position at $18 \mathrm{ML}$ taken as a reference for relaxed bcc Fe without contraction normal to the surface, the shift of the intensity peak from 25.0 to $20.4 \mathrm{eV}$ between 1 and $12 \mathrm{ML}$ establishes a vertical contraction that decreases from $14.2 \%$ to $6.6 \%$ in this thickness range. The persistence of a strong contraction to large thickness is enabled by a high defect concentration as seen by the strong decrease of the spot to background intensity ratio in Fig. 3(b). Nevertheless, the strain energy stored in the metastable layer apparently becomes so large that it breaks up just after $12 \mathrm{ML}$, exposing part of the bare substrate or 1 ML covered substrate with its strong (00) intensity peak visible at about 14.8$14.0 \mathrm{eV}$ between 12.4 and 14.2 ML. Further deposition causes the now highly disordered Fe layer to recrystallize into separated very small grains with bulk normal layer spacing and possibly varying height. The roughness of the layer and small grain size both contribute to the strong diffuse scattering. We believe that this microstructure only produces significant diffraction at the specular (00) spot position, while we attribute the persistent but strongly diminishing diffraction intensity at the original integer order diffraction spot positions to a diminishing component of the film that has yet to relax to bulklike bcc structure.

Finally, we note that our new analysis of the LEED patterns reported in Ref. 18 indeed shows that they are incompatible with fcc Fe. Rather, they are fully consistent with bcc Fe, which is laterally compressed by $3.4 \%$ compared to bulk Fe between 6 and $12 \mathrm{ML}$, forming a 7:8 coincidence lattice with the substrate. Above $12 \mathrm{ML}$ the lateral periodicity seen in LEED in Ref. 18 agrees within the limits of error with that of bulk bcc Fe(111). Nevertheless, the structural modifications reported to take place in $\mathrm{Fe} / \mathrm{W}(111)$ in Ref. 18 are mirrored by modifications of structure revealed here with increasing thickness (Fig. 2).

\section{B. Magnetic properties}

We observed large magnetic domains, far exceeding the image field of view, from the onset of magnetization at room temperature (6 ML) up to about $12 \mathrm{ML}$. The inplane magnetization direction observed here in this thickness range and at larger thicknesses is consistent with transverse spin polarization of field emitted electrons from Fe-coated $\mathrm{W}(111)$ tips. ${ }^{19}$ In that work, numerous transverse polarization directions were observed with a tendency for $30 \mathrm{deg}$ (12-fold) intervals. This observation was attributed to the interplay between magnetocrystalline anisotropy and step-induced effects arising from the tip morphology. Similarly, the single domain morphology observed here may be due to the influence of atomic surface steps, arising from a small sample miscut, although diffraction spot streaking that would be expected from significant miscut was not observed. This effect may simply have been washed out by the broadening of diffraction spots arising from the underlying roughness of the W(111) substrate noted in Sec. II.

Changes of the exchange asymmetry vs energy $A_{\mathrm{ex}}(E)$ up to $12 \mathrm{ML}$ are indicative of corresponding changes in the spin polarized electronic band structure above the vacuum level. These changes may be induced by the gradual vertical relaxation of the laterally strained bcc Fe layer, which is indicated by the $I(V)$ measurements (Fig. 2). On the other hand, the change from monodomain to multidomain structure at about $12 \mathrm{ML}$ coincides with much more dramatic changes of the $A_{\mathrm{ex}}(E)$ characteristics [Fig. 6(a)]. With the insight provided by diffraction intensity measurements (Figs. 2 and 3 ), we understand these changes to be linked to significant restructuring of the Fe film to relaxed although highly defective bcc Fe structure and film microstructure.

The most striking behavior is the complete loss of magnetic asymmetry for film thickness between 8 and $9 \mathrm{ML}$ at room temperature (Fig. 5), confirmed by the absence of asymmetry at every incident electron energy and incident beam polarization direction (Fig. 6). One possible explanation is that the film is nonmagnetic, although complete suppression of magnetic moments seems unlikely. Temperature dependent measurements in the 6-8 ML range (Figs. 7 and 8 ) suggest that the Curie temperature may decrease below room temperature in the thickness range of the ferromagnetic gap. Further work is needed to confirm this speculation. This might occur due to the interplay between structure and magnetism. In particular, vertical rearrangements with increasing thickness may tend to suppress magnetic ordering at room temperature, in competition with an increasing trend of the Curie temperature with thickness due to finite size effects. ${ }^{35}$ A significant change of the spin polarized band structure across this gap is evident in the energy dependence of magnetic asymmetry (Fig. 6), from single to double peak, that may have its origin in structural rearrangement across the gap.

Interestingly, a similar ferromagnetic thickness gap behavior was observed in spin polarized LEED measurements of exchange asymmetry for Fe films on the W(110) surface. ${ }^{37}$ The exchange asymmetry was suppressed in the coverage range between 1.20 and $1.48 \mathrm{ML}$. A very similar trend of the Curie temperature vs thickness was also seen between the onset thickness for ferromagnetic order and the start of the gap. This behavior was attributed to an antiferromagnetic interaction between double layer islands, mediated by the intervening $\mathrm{Fe}$ monolayer and the $\mathrm{W}$ substrate, which frustrates long range ferromagnetic order. Observations of film morphology with scanning tunneling microscopy revealed island morphology that supported the physical explanation of the observed gap. Similar frustration of long range magnetic order, rooted in film morphology, may also be responsible for the observed gap in Fe/W(111). Alternatively, the correlation with morphology may simply be caused by competing shape, magnetocrystalline, and interface anisotropies. 


\section{CONCLUSIONS}

We observe that $\mathrm{Fe}$ grows on the W(111) surface at room temperature as a vertically contracted and laterally (tensile) strained bcc layer that is well ordered initially but gradually relaxes vertically and develops increasing disorder with increasing thickness. The strain energy stored in the metastable layer becomes so large by $12 \mathrm{ML}$ thickness that it breaks up and forms small grains of relaxed bcc Fe. This behavior agrees with the initial pseudomorphic growth at room temperature up to $6 \mathrm{ML}$ on the $\mathrm{W}(111)$ surface reported previously but contrasts with the interpretation of earlier LEED observations of the formation of fcc Fe films above 6 ML. ${ }^{18}$ Our reanalysis of the previously published LEED patterns reveals that superstructure diffraction spots, attributed earlier to fcc Fe, are due instead to bcc Fe that is laterally contracted initially and relaxed at larger thicknesses. The disparity between LEED observations reported here and previously ${ }^{18}$ is attributed to the different deposition conditions employed in the two investigations. We believe that the continuous deposition method employed here leads to metastable film structure and disorder, whereas interrupted film growth by incremental deposition as carried out previously may be necessary to suppress disorder and obtain films with better long range order. However, the influence of residual gas cannot be ruled out.

Changes of the magnetic exchange asymmetry in elastic spin-polarized electron scattering are also observed with increasing film thickness that may be due to changes in the spin polarized electron band structure above the vacuum level. The transition from a large monodomain structure with uniaxial magnetization to a granular multidomain structure between about 12 and $15 \mathrm{ML}$ correlates with very significant changes of exchange asymmetry, film breakup, and appearance of relaxed bcc Fe. Further work is needed to develop a more comprehensive and possibly quantitative understanding of the relationship between changes in magnetic exchange asymmetry and the structural rearrangements reported here. The origin of a striking ferromagnetic thickness gap between 8 and 9 ML thickness is not clear, but could be related to the vertical relaxation of the film through finite size effects. The observed gap also bears a striking resemblance to behavior observed earlier in Fe/W(110) films, which was attributed to morphological frustration to long range order. Scanning tunneling microscopy measurements of film morphology would help to identify if a similar frustration could be responsible for the gap in $\mathrm{Fe} / \mathrm{W}(111)$.

\section{ACKNOWLEDGMENTS}

We gratefully acknowledge funding from the Hong Kong Research Grants Council under Grant 600111 and from the Polish Ministry of Science and Higher Education under Grant No. N N202 174539. *phaltman@ust.hk

${ }^{1}$ J. Kübler, Phys. Lett. A 81, 81 (1981).

${ }^{2}$ C. S. Wang, B. M. Klein, and H. Krakauer, Phys. Rev. Lett. 54, 1852 (1985).

${ }^{3}$ F. J. Pinski, J. Staunton, B. L. Gyorffy, D. D. Johnson, and G. M. Stocks, Phys. Rev. Lett. 56, 2096 (1986)

${ }^{4}$ V. L. Moruzzi, P. M. Marcus, K. Schwarz, and P. Mohn, Phys. Rev. B 34, 1784 (1986).

${ }^{5}$ Y. Tsunoda, J. Phys.: Condens. Matter 1, 10427 (1989).

${ }^{6}$ O. N. Mryasov, V. A. Gubanov, and A. I. Liechtenstein, Phys. Rev. B 45, 12330 (1992).

${ }^{7}$ M. Uhl, L. M. Sandratskii, and J. Kübler, Phys. Rev. B 50, 291 (1994).

${ }^{8}$ E. Sjöstedt and L. Nordström, Phys. Rev. B 66, 014447 (2002).

${ }^{9}$ D. Tian, F. Jona, and P. M. Marcus, Phys. Rev. B 45, 11216 (1992).

${ }^{10}$ J. Shen, M. Klaua, P. Ohresser, H. Jenniches, J. Barthel, Ch. V. Mohan, and J. Kirschner, Phys. Rev. B 56, 11134 (1997).

${ }^{11}$ J. Thomassen, F. May, B. Feldmann, M. Wuttig, and H. Ibach, Phys. Rev. Lett. 69, 3831 (1992).

${ }^{12}$ D. Qian, X. F. Jin, J. Barthel, M. Klaua, and J. Kirschner, Phys. Rev. Lett. 87, 227204 (2001).

${ }^{13}$ K. L. Man, W. L. Ling, S. Y. Paik, H. Poppa, M. S. Altman, and Z. Q. Qiu, Phys. Rev. B 65, 024409 (2001).

${ }^{14}$ J. Giergiel, J. Shen, J. Woltersdorf, A. Kirilyuk, and J. Kirschner, Phys. Rev. B 52, 8528 (1995).

${ }^{15}$ A. Biedermann, M. Schmid, and P. Varga, Phys. Rev. Lett. 86, 464 (2001).

${ }^{16}$ D. Spišák and J. Hafner, Phys. Rev. Lett. 88, 056101 (2002).

${ }^{17}$ A. Biedermann, W. Rupp, M. Schmid, and P. Varga, Phys. Rev. B 73, 165418 (2006).
${ }^{18}$ J. Kołaczkiewicz and E. Bauer, Surf. Sci. 420, 157 (1999).

${ }^{19}$ Y. Niu and M. S. Altman, Surf. Sci. 604, 1055 (2010).

${ }^{20}$ R. Bryl and M. S. Altman, J. Appl. Phys. 94, 4670 (2003).

${ }^{21}$ K. L. Man, R. Zdyb, S. F. Huang, T. C. Leung, C. T. Chan, E. Bauer, and M. S. Altman, Phys. Rev. B 67, 184402 (2003).

${ }^{22}$ T. Duden, R. Zdyb, M. S. Altman, and E. Bauer, Surf. Sci. 480, 145 (2001).

${ }^{23}$ J. Guan, R. A. Campbell, and T. E. Madey, Surf. Sci. 341, 311 (1995).

${ }^{24}$ E. Bauer, Rep. Prog. Phys. 57, 895 (1994).

${ }^{25}$ M. S. Altman, J. Phys.: Condens. Matter 22, 084017 (2010).

${ }^{26}$ R. Zdyb and E. Bauer (to be published)

${ }^{27}$ A. B. Pang, Th. Müller, M. S. Altman, and E. Bauer, J. Phys.: Condens. Matter 21, 314006 (2009).

${ }^{28}$ K. L. Man and M. S. Altman, Phys. Rev. B 84, 235415 (2011).

${ }^{29}$ T. Duden and E. Bauer, Rev. Sci. Instrum. 66, 2861 (1995).

${ }^{30}$ C. M. Yim, K. L. Man, Xudong Xiao, and M. S. Altman, Phys. Rev. B 78, 155439 (2008).

${ }^{31}$ Y. Murata, E. Starodub, B. B. Kappes, C. V. Ciobanu, N. C. Bartelt, K. F. McCarty, and S. Kodambaka, Appl. Phys. Lett. 97, 143114 (2010).

${ }^{32}$ V. N. Strocov, P. O. Nilsson, R. Claessen, F. Aryasetiawan, P. Blaha, J.-M. Themlin, G. Nicolay, and S. Hüfner, Surf. Rev. Lett. 9, 1281 (2002).

${ }^{33}$ J. I. Flege, A. Meyer, J. Falta, and E. E. Krasovskii, Phys. Rev. B 84, 115441 (2011).

${ }^{34}$ The vertical layer spacing of $\mathrm{W}(111)$ is $1.82 \AA$. The vertical layer spacings of 2.04 and $1.37 \AA$ for lattice-matched fcc Fe(111)/W(111) and ps bcc $\mathrm{Fe}(111) / \mathrm{W}(111)$, respectively, are obtained after taking into account the lateral strain through the conservation of 
atomic volume with respect to unstrained bulk counterparts. In the estimation of the layer spacing of lattice-matched fcc Fe(111)/ $\mathrm{W}(111)$, the next nearest neighbor distance of the bulk Fe(111) layer $(4.42 \AA)$ is assumed to be expanded to match the nearest neighbor distance of W(111) (4.47 $\AA$ ).

${ }^{35}$ U. Gradmann, in Handbook of Magnetic Materials, edited by K. H. J. Buschow (Elsevier, Amsterdam, 1993).
${ }^{36}$ H. J. Elmers and J. Hauschild, Surf. Sci. 320, 134 (1994).

${ }^{37}$ H. J. Elmers, J. Hauschild, H. Fritzsche, G. Liu, U. Gradmann, and U. Köhler, Phys. Rev. Lett. 75, 2031 (1995).

${ }^{38}$ R. Zdyb, T. O. Menteş, A. Locatelli, M. A. Niño, and E. Bauer, Phys. Rev. B 80, 184425 (2009).

${ }^{39}$ J. Kołaczkiewicz (private communication). 